\title{
Semilinear Parabolic Equations with Prescribed Energy
}

\author{
by \\ Bei $\mathrm{Hu}^{1}$ and Hong-Ming Yin \\ Department of Mathematics \\ University of Notre Dame \\ Notre Dame, IN 46556, USA.
}

\begin{abstract}
In this paper we study the following reaction-diffusion equation $u_{t}=\Delta u+$ $f(u, k(t))$ subject to appropriate initial and boundary conditions, where $f(u, k(t))=$ $u^{p}-k(t)$ or $k(t) u^{p}$ with $p>1$ and $k(t)$ is an unknown function. An additional energy type condition is imposed in order to find the solution $u(x, t)$ and $k(t)$. This type of problem is frequently encountered in nuclear reaction process, where the reaction is known to be very strong, but the total energy is controlled. It is shown that the solution blows up in finite time for the first class of function $f$ for some initial data. For the second class of function $f$, the solution blows up in finite time if $p>n /(n-2)$ while it exists globally in time if $1<p<n /(n-2)$, no matter how large the initial value is. Partial results are generalized into the case where $f(u, k(t))$ appears on the boundary.
\end{abstract}

Key Words and Phrases: Inverse Problems, Diffusion with Strong Reaction, Global Solvability, Finite time Blowup .

AMS(MOS) Subject Classification: 35K57, 35B05.

\footnotetext{
${ }^{1}$ This author is partially supported by National Science Foundation Grant DMS 92-24935
} 


\section{Introduction}

Consider a chemical reaction-diffusion process, where it is known that the reaction is very strong, say like $u^{p}$ with $p>1$, but the rate with respect to this power is unknown, say $k(t)$, a function of $t$. On the other hand, the total energy is controlled in the system in order to prevent the blowup phenomenon. That is

$$
\int_{\Omega} u(x, t) d x=g(t)
$$

This leads to an inverse problem where one needs to find the solution $u(x, t)$ as well as the coefficient of the strong reaction. Another model arising from the nuclear science is that the growth of temperature is known to be very fast like $u^{p}$, but some absorption catalystic material is put into the system in such a way that the total mass is conserved. These two models leads us to consider the following parabolic inverse problem: Find $u(x, t)$ and $k(t)$ such that

$$
\begin{aligned}
& u_{t}-\Delta u=f(u, k(t)) \text { for }(x, t) \in Q_{T}=\Omega \times(0, T] \\
& \frac{\partial u}{\partial \nu}(x, t)=0 \text { for }(x, t) \in S_{T}=\partial \Omega \times[0, T] \\
& u(x, 0)=u_{0}(x) \text { for } x \in \Omega,
\end{aligned}
$$

where $\Omega$ is a bounded domain in $R^{n}$ with a smooth boundary $S=\partial \Omega$ and $\nu$ is the outward normal on $S$. An additional energy condition is prescribed by

$$
\int_{\Omega} u(x, t) d x=g(t), \quad t \geq 0 .
$$

The above mathematical problem can also be used to model other phenomena in population dynamics and biological sciences where the total mass is often conserved or known, but the growth of a certain cell is known to be of some form. The research on the well-posedness of a parabolic inverse problem goes back to 60 's (see the references in [4]). Since then, considerable progress has been made regarding to various inverse problems for parabolic equations. The reader can find a large number of references in [4]-[6] and the recent proceedings [9]. The essential difference between the previous inverse problems and the current one is that the 
solution of (1.1)-(1.4) may blow up; this fact is well known when $k(t)$ is given. On the other hand, since our $k(t)$ is given in terms of the solution, the problem may have a global solution because of the stabilizing factor $k(t)$. The natural question is whether or not the stabilizing factor is strong enough to prevent the blowup. We shall study this problem in the present work. We shall only discuss two classes of function $f(u, k(t))$, namely,

$$
f(u, k(t))=u^{p}-k(t) \quad \text { or } k(t) u^{p}
$$

When the function $k(t)>0$ is given, there are a lot of papers (cf. [2], [10], [14], [17] and the references therein ) dealing with various qualitative properties such as finite time blowup, blowup rate, blowup set. When $f(u, k(t))=u^{p}-k(t)$ or $k(t) u^{p}$ with an unknown $k(t)$, it is not difficult to see that the condition (1.4) and the equation (1.1) imply

$$
k(t)=\frac{1}{|\Omega|}\left(\int_{\Omega} u^{p} d x-g^{\prime}(t)\right), \quad \text { or } \quad \frac{g^{\prime}(t)}{\int_{\Omega} u^{p} d x .}
$$

Hence the reaction term in (1.1) can be written as

$$
f=u^{p}-\frac{1}{|\Omega|}\left(\int_{\Omega} u^{p} d x-g^{\prime}(t)\right), \quad \text { or } \quad \frac{g^{\prime}(t) u^{p}}{\int_{\Omega} u^{p} d x} .
$$

In general, it is not clear which term can dominate the reaction. As there is a nonlocal integral term in the equation (1.1), the powerful comparison principle is invalid. We prove by applying the energy method that, for the first class of the function $f$, the solution will blow up in finite time for a class of initial data. For the second case, one may also believe that the solution will blow up in finite time. Surprisingly, it turns out that the finite time blowup or global existence depends upon the exponent $p$ and the space dimension $n$. It will be seen in Section 3 that the solution exists globally if $p<n /(n-2)$, no matter how large the initial data are. On the other hand, the solution will blow up in finite time if $p>n /(n-2)$, provided that initial data satisfy some conditions. This is quite different from a regular reaction-diffusion equation (cf. [2], [10], [14], etc.). We shall mention 
that diffusion equations with nonlocal reactions has been considered by a number of authors (cf. [1], [7]-[8], [17], etc.). However, none of those deals with similar problems to (1.1)-(1.4), since the energy in these previous problems blows up in finite time. More recently, the authors of [3] studied the problem (1.1)-(1.4) with $f(u, k(t))=u^{2}-\int_{0}^{1} u^{2} d x$ in one space dimension. Blowup is proved for some special initial value and the blowup rate is also discussed. The argument for proving the blowup property is obviously not suitable for the present situation.

The paper is organized in the following way. In Section 2, we study the problem with $f(u, k(t))=u^{p}-k(t)$. In Section 3, we show the global existence for the case where $f(u, k(t))=k(t) u^{p}$ with $p<n /(n-2)$. We also prove the global existence for $p=n /(n-2)$ when the initial value is large enough. In Section 4 , we consider the case with $p>n /(n-2)$ and prove that the solution blows up in finite time for suitable radially symmetric initial data. Section 5 deals with the case where $f(u, k(t))$ occurs on the boundary.

\section{Blowup for $f(u, k(t))=u^{p}-k(t)$}

Throughout this paper, $C$ denotes generic constants, unless otherwise indicated. Mathematically, we do not require that $u(x, t)$ is nonnegative. Therefore we shall use $|u|^{p-1} u$ instead of $u^{p}$. For some technical reasons, the argument below is valid when $g(t)=$ constant, say 1 . This is the case in some applications. The equation (1.1)-(1.4) can now be rewritten as follows (with $g(t)=1$ ):

$$
\begin{aligned}
& u_{t}=\Delta u+|u|^{p-1} u-k(t) \text { for } x \in \Omega, t>0, \\
& \frac{\partial u}{\partial \nu}=0 \text { for } x \in \partial \Omega, t>0 \\
& u(x, 0)=u_{0}(x) \text { for } x \in \Omega .
\end{aligned}
$$

where

$$
k(t)=\frac{1}{|\Omega|}\left(\int_{\Omega}|u|^{p-1} u d x\right) .
$$

The following conditions on the data are assumed throughout this section: $\mathbf{H}(\mathbf{A}) \quad u_{0}(x) \in C^{3}(\bar{\Omega}) \quad$ and $\int_{\Omega} u_{0}(x) d x=1$. 
The existence and uniqueness of this system for small $t$ is clear, by the standard theory of parabolic estimates and contraction mapping principle. It is also clear that the solution can be extended in $t$ direction, as long as the $L^{\infty}$ norm of the solution remains finite.

Theorem 2.1: The solution of (2.1) blows up in finite time if $p>1$ and

$$
-\frac{1}{2} \int_{\Omega}\left|\nabla u_{0}\right|^{2} d x+\frac{1}{p+1} \int_{\Omega}\left|u_{0}\right|^{p+1} d x
$$

is suitably large.

Proof: The proof is based convexity argument (see Levine and Payne [15]). We multiply the equation by $u$ and $u_{t}$, respectively, and then integrate over $\Omega$, to obtain

$$
\begin{aligned}
& \frac{d}{d t}\left(\frac{1}{2} \int_{\Omega} u^{2} d x\right)+\int_{\Omega}|\nabla u|^{2} d x=\int_{\Omega}|u|^{p+1} d x-\frac{1}{|\Omega|} \int_{\Omega}|u|^{p-1} u d x \\
& \int_{\Omega} u_{t}^{2} d x+\frac{d}{d t} \int_{\Omega} \frac{1}{2}|\nabla u|^{2} d x=\frac{d}{d t}\left(\frac{1}{p+1} \int_{\Omega}|u|^{p+1} d x\right)
\end{aligned}
$$

Let

$$
J(t)=-\frac{1}{2} \int_{\Omega}|\nabla u|^{2} d x+\frac{1}{p+1} \int_{\Omega}|u|^{p+1} d x
$$

The identity (2.3) gives

$$
J^{\prime}(t)=\int_{\Omega} u_{t}^{2} d x \geq 0
$$

It follows that

$$
J(t)=J(0)+\int_{0}^{t} \int_{\Omega} u_{t}^{2} d x d t
$$

Introduce a new function

$$
I(t)=\int_{0}^{t} \int_{\Omega} u^{2} d x d t+A+B t^{2}
$$

where $A$ and $B$ are two constants to be specified later. Clearly

$$
I^{\prime}(t)=\int_{\Omega} u^{2} d x+2 B t, \quad I^{\prime \prime}(t)=\frac{d}{d t} \int_{\Omega} u^{2} d x+2 B .
$$


By the identity (2.2),

$$
\begin{aligned}
I^{\prime \prime}(t) & =\frac{d}{d t} \int_{\Omega} u^{2} d x+2 B \\
& =-2 \int_{\Omega}|\nabla u|^{2} d x+2 \int_{\Omega}|u|^{p+1} d x-\frac{2}{|\Omega|} \int_{\Omega}|u|^{p-1} u d x+2 B .
\end{aligned}
$$

We claim that there exists a constant $\delta>0$ such that

$$
I^{\prime \prime}(t) \geq 4(1+\delta) J(t)
$$

Indeed, the desired the inequality is equivalent to

$$
\begin{gathered}
-2 \int_{\Omega}|\nabla u|^{2} d x+2 \int_{\Omega}|u|^{p+1} d x-\frac{2}{|\Omega|} \int_{\Omega}|u|^{p-1} u d x+2 B \\
\geq 4(1+\delta)\left[-\frac{1}{2} \int_{\Omega}|\nabla u|^{2} d x+\frac{1}{p+1} \int_{\Omega}|u|^{p+1} d x\right] .
\end{gathered}
$$

Now Hölder's and Young's inequalities imply that for any $\delta_{1}>0$

$$
\int_{\Omega}|u|^{p} d x \leq \delta_{1} \int_{\Omega}|u|^{p+1} d x+C\left(\delta_{1},|\Omega|\right) .
$$

As $p>1$, we choose $\delta$ and $\delta_{1}$ to be small enough such that

$$
2-\frac{4(1+\delta)}{1+p}-\delta_{1}>0 .
$$

Then we have the desired claim if we simply take $B \geq C\left(\delta_{1},|\Omega|\right)$.

Clearly

$$
I^{\prime}(t)=\int_{\Omega} u^{2} d x+2 B t=2 \int_{0}^{t} \int_{\Omega} u u_{t} d x d t+\int_{\Omega} u_{0}^{2} d x+2 B t .
$$

It follows that, for any $\varepsilon>0$,

$$
I^{\prime}(t)^{2} \leq 4(1+\varepsilon) \int_{0}^{t} \int_{\Omega} u^{2} d x d t \int_{0}^{t} \int_{\Omega} u_{t}^{2} d x d t+\left(1+\frac{1}{\varepsilon}\right)\left[\int_{\Omega} u_{0}^{2} d x+2 B t\right]^{2} .
$$

Combining the above estimates, we find that, for $\alpha>0$,

$$
\begin{aligned}
I^{\prime \prime}(t) I(t)- & (1+\alpha) I^{\prime}(t)^{2} \\
\geq 4(1+ & \delta)\left[J(0)+\int_{0}^{t} \int_{\Omega} u_{t}^{2} d x d t\right]\left[\int_{0}^{t} \int_{\Omega} u^{2} d x d t+A+B t^{2}\right] \\
& -(1+\alpha)\left[4(1+\varepsilon) \int_{0}^{t} \int_{\Omega} u^{2} d x d t \int_{0}^{t} \int_{\Omega} u_{t}^{2} d x d t\right] \\
& -(1+\alpha)\left(1+\frac{1}{\varepsilon}\right)\left[\int_{\Omega} u_{0}^{2} d x+2 B t\right]^{2} .
\end{aligned}
$$


Now we choose $\varepsilon$ and $\alpha$ to be small enough such that

$$
1+\delta \geq(1+\alpha)(1+\varepsilon)
$$

If

$$
J(0) \geq 4(1+\alpha)\left(1+\frac{1}{\varepsilon}\right) B
$$

and $A$ is chosen to be large enough, then we have

$$
I^{\prime \prime}(t) I(t)-(1+\alpha) I^{\prime}(t)^{2} \geq 0 .
$$

It follows (cf. [15]) that $\int_{0}^{t} \int_{\Omega} u^{2} d x d t$ will blow up in a finite time $T^{*}$, if the solution exists in $\left(0, T^{*}\right)$.

Remark 2.1: When $u_{0}(x)=M$ is a constant, then $u(x, t)=M$ is always a solution. In this case, the proof is invalid since in the inequality (2.6) the constant $B$ depends on $|\Omega|$, but the condition H(A) implies $M|\Omega|=1$. Consequently, $B$ depends on $M$, so do the other constants in the proof. Therefore, it is impossible to choose $J(0)$ to satisfy the desired inequality (2.10).

\section{Global Existence for $f(u, k(t))=k(t) u^{p}$}

For simplicity, we shall assume that $g^{\prime}(t)=1$ in Sections 3 and 4 . It will be seen that the results can be immediately carried over for a general function $g(t)>0$ with $g^{\prime}(t)>0$ in this section. In this case the problem (1.1)-(1.4) is equivalent to the following:

$$
\begin{aligned}
& u_{t}=\Delta u+k(t) u^{p} \quad \text { for } x \in \Omega, t>0, \\
& \frac{\partial u}{\partial \nu}=0 \text { for } x \in \partial \Omega, t>0, \\
& u(x, 0)=u_{0}(x) \text { for } x \in \Omega .
\end{aligned}
$$

where

$$
k(t)=\frac{1}{\int_{\Omega} u^{p}(x, t) d x} .
$$


We shall assume throughout this section that $u_{0}(x)$ is smooth, $u_{0}(x)>0$ on $\bar{\Omega}$, and satisfies the compatibility condition $\frac{\partial u_{0}}{\partial \nu}=0$ on $\partial \Omega$ and $\int_{\Omega} u_{0}(x) d x=m>0$.

By the standard theory of parabolic estimates and contraction mapping principle, the existence and uniqueness of this system for small $t$ is guaranteed. The solution can be extended in $t$ direction, as long as the solution remains finite. Since we assume that the initial data are positive, the solution $u(x, t)$ is positive, by the maximum principle.

Theorem 3.1: Suppose that $1<p<n /(n-2)$ for $n \geq 3$ and $1<p<\infty$ when $n=1$ or 2 . Then there exists a unique global solution to the system, namely, the solution exists for all $t \in[0, \infty)$.

Proof: The proof here is for $n \geq 3$. The proof for $n=1$ and $n=2$ can be obtained with obvious modifications. We fix a large $\beta$. Without loss of generality, we assume that $|\Omega|=1$. For any $0<a<1$, and $\frac{1}{q}+\frac{1}{q^{*}}=1$, we have

$$
\int_{\Omega} u^{p+\beta} d x \leq\left(\int_{\Omega} u^{(p+\beta) a q} d x\right)^{1 / q}\left(\int_{\Omega} u^{(p+\beta)(1-a) q^{*}} d x\right)^{1 / q^{*}} .
$$

There are two free parameters $a$ and $q$ to be determined. We first let

$$
(p+\beta) a q=(\beta+1) \frac{n}{n-2} .
$$

If $q=n /(n-2)$ (it is clear that $0<a<1$ with this choice of $q$, as $p>1$ ), then

$$
(p+\beta)(1-a) q^{*}=\frac{n}{2}(p-1)<p .
$$

However, the choice $q=n /(n-2)$ will not be good enough in our proof. Since we have a strict inequality in (3.4) when $q=n /(n-2)$, we can take $q>n /(n-2)$ and $q-n /(n-2) \ll 1$ so that $(3.4)$ is still valid for this particular choice of $q$. Using Hölder's inequality again, we obtain, (recalling that $|\Omega|=1$ ),

$$
\begin{aligned}
\int_{\Omega} u^{p+\beta} d x & \leq\left(\int_{\Omega} u^{(\beta+1) \frac{n}{n-2}} d x\right)^{1 / q}\left(\int_{\Omega} u^{(p+\beta)(1-a) q^{*}} d x\right)^{1 / q^{*}} \\
& \leq\left(\int_{\Omega} u^{(\beta+1) \frac{n}{n-2}} d x\right)^{1 / q}\left(\int_{\Omega} u^{p} d x\right)^{(p+\beta)(1-a) / p}
\end{aligned}
$$


Next, multiplying the equation by $u^{\beta}$ and integrating over $\Omega$, we obtain

$$
\left(\int_{\Omega} \frac{1}{\beta+1} u^{\beta+1} d x\right)_{t}+\beta \int_{\Omega} u^{\beta-1}|\nabla u|^{2} d x=\frac{\int_{\Omega} u^{p+\beta} d x}{\int_{\Omega} u^{p} d x} .
$$

Integrating the equation, we immediately get

$$
\int_{\Omega} u(x, t) d x=\int_{\Omega} u_{0}(x) d x+t .
$$

Hence by Hölder's inequality and the fact $|\Omega|=1$,

$$
\int_{\Omega} u^{p} d t \geq\left(\int_{\Omega} u_{0}(x) d x+t\right)^{p} \geq\left(\int_{\Omega} u_{0}(x) d x\right)^{p}=m^{p}>0 .
$$

Using (3.4), (3.5), (3.6), (3.7), we obtain

$$
\left(\int_{\Omega} \frac{1}{\beta+1} u^{\beta+1} d x\right)_{t}+\frac{4 \beta}{(\beta+1)^{2}} \int_{\Omega}\left|\nabla u^{(\beta+1) / 2}\right|^{2} d x \leq C\left(\int_{\Omega} u^{(\beta+1) \frac{n}{n-2}} d x\right)^{1 / q} .
$$

If we define $v=u^{(\beta+1) / 2}$, then

$$
\left(\frac{1}{\beta+1} \int_{\Omega} v^{2} d x\right)_{t}+\frac{4 \beta}{4(\beta+1)^{2}} \int_{\Omega}|\nabla v|^{2} d x \leq C\left(\int_{\Omega} v^{\frac{2 n}{n-2}} d x\right)^{1 / q} .
$$

Now we use the following (elliptic) Sobolev's embedding theorem (cf. [16]) in the above inequality.

$$
\|w\|_{L^{r}(\Omega)} \leq C\left(\|\nabla w\|_{L^{2}(\Omega)}+\|w\|_{L^{2}(\Omega)}\right), \quad\left(r=\frac{2 n}{n-2}\right) .
$$

Since $q>n /(n-2)$, we obtain

$$
\left(\int_{\Omega} v^{2} d x\right)_{t}+\int_{\Omega}|\nabla v|^{2} d x \leq C_{\beta}\left(\int_{\Omega} v^{2} d x+1\right) .
$$

By Gronwall's inequality, we conclude that the right-hand-side of the above inequality is bounded. Therefore

$$
\begin{gathered}
\left(\int_{\Omega} u^{\beta+1} d x\right)_{t}+\left(\int_{\Omega} u^{(\beta+1) \frac{n}{n-2}} d x\right)^{\frac{n-2}{n}} \leq C_{\beta}, \\
\sup _{0 \leq t \leq T}\left(\int_{\Omega} u^{\beta+1} d x\right)+\int_{0}^{t}\left(\int_{\Omega} u^{(\beta+1) \frac{n}{n-2}} d x\right)^{\frac{n-2}{n}} d t \leq C_{\beta} .
\end{gathered}
$$


It follows that $u$ is bounded in $L^{\beta+1}$ for any finite $\beta$. Thus the $L^{p}$ estimates (cf. [13]) implies that $u$ is in $W_{(\beta+1) / p}^{2,1}\left(Q_{T}\right)$ for any $\beta$. Therefore if we take $(\beta+1) / p>$ $(n+2) / 2$, then by the Sobolev's embedding theorem, $u$ is Hölder continuous, for $0 \leq t \leq T$, for any $T>0$. It is then easily seen that $u \in C^{\infty}\left(Q_{T}\right)$. Thus we obtain a global solution.

We now turn to the case where $p$ is equal to the critical number $n /(n-2)$. The above proof with $q=n /(n-2)$ gives (recalling that $|\Omega|=1$ )

$$
\begin{aligned}
\left(\int_{\Omega} u^{\beta+1} d x\right)_{t}+\int_{\Omega}\left|\nabla u^{(\beta+1) / 2}\right|^{2} d x & \leq C_{\beta} \frac{\left(\int_{\Omega} u^{(\beta+1) \frac{n}{n-2}} d x\right)^{\frac{n-2}{n}}}{\left(\int_{\Omega} u^{p} d x\right)^{1 / p}} \\
& \leq C_{\beta} \frac{\left(\int_{\Omega} u^{(\beta+1) \frac{n}{n-2}} d x\right)^{\frac{n-2}{n}}}{t+\int_{\Omega} u_{0}(x) d x},
\end{aligned}
$$

where at the final step we have used the inequality (3.7). By Sobolev's embedding, the numerator in the right-hand side of (3.11) can be dominated by

$$
C\left(\int_{\Omega} u^{\beta+1} d x+\int_{\Omega}\left|\nabla u^{(\beta+1) / 2}\right|^{2} d x\right)
$$

We now fix $\beta$ in (3.11) such that $(\beta+1) / p>(n+2) / 2$ and choose $\int_{\Omega} u_{0}(x) d x$ to be large enough, then we have the same estimates as (3.10). Consequently, we proved

Theorem 3.2: Suppose that $p=n /(n-2)$ for $n \geq 3$. Then the solution exists for all $t \in[0, \infty)$, provided $\int_{\Omega} u_{0}(x) d x$ is large enough.

\section{Blowup of solutions for $f(u, k(t))=k(t) u^{p}$}

In this section we shall construct a solution which blows up in finite time when $p>n /(n-2)$.

Theorem 4.1: Suppose that $p>n /(n-2)$. Then there exists a radially symmetric 
initial datum $u_{0}(x)$ (with $\Omega=B_{1}(0)$ ) such that the corresponding solution $u(x, t)$ of (3.1) blows up in finite time at $x=0$. Furthermore, $x=0$ is the only blowup point.

Proof: Let

$$
\varphi(r)=\left\{\begin{array}{l}
\frac{1}{r^{\alpha}} \text { for } \delta<r \leq 1, \\
\frac{1}{\delta^{\alpha}}\left(1+\frac{\alpha}{2}\right)-\frac{\alpha}{2 \delta^{\alpha+2}} r^{2} \quad \text { for } 0 \leq r \leq \delta,
\end{array}\right.
$$

where we choose

$$
\alpha=\frac{2}{p-1} .
$$

It is clear that $\varphi(r)$ is in $C^{1}[0,1] \cap C^{\infty}([0, \delta) \cup(\delta, 1])$, and $\varphi^{\prime}(0)=0$ and $\varphi^{\prime}(r) \leq 0$. A direct calculation shows that

$$
\begin{aligned}
\int_{0}^{1} n \omega_{n} \varphi^{p}(r) r^{n-1} d r & =\int_{0_{0}}^{1} n \omega_{n} r^{n-1-p \alpha} d r+\sigma_{1}(\delta) \\
& =\frac{n-\alpha p}{n-\alpha}+\sigma_{1}(\delta)
\end{aligned}
$$

where

$$
\sigma_{1}(\delta)=-\int_{0}^{\delta} n \omega_{n} r^{n-1-p \alpha} d r+\int_{0}^{\delta} n \omega_{n}\left[\frac{1}{\delta^{\alpha}}\left(1+\frac{\alpha}{2}\right)-\frac{\alpha}{2 \delta^{\alpha+2}} r^{2}\right]^{p} r^{n-1} d r=O\left(\delta^{n-\alpha p}\right),
$$

and $\omega_{n}$ is the surface area of unit ball in $R^{n}$. Thus $\sigma_{1}(\delta) \rightarrow 0$ as $\delta \rightarrow 0+$, since $p>n /(n-2)$. From our choice of $\alpha$, we find that for each $\delta \in(0,1)$,

$$
\begin{aligned}
\varphi_{r r}+\frac{n-1}{r} \varphi_{r} & =\frac{\alpha}{r^{\alpha+2}}((\alpha+1)-(n-1)) \\
& =-\alpha((n-2)-\alpha) \varphi^{p} \text { for } \delta \leq r<1,
\end{aligned}
$$

and

$$
\begin{aligned}
\varphi_{r r}+\frac{n-1}{r} \varphi_{r} & =-\frac{n \alpha}{\delta^{\alpha+2}}=-n \alpha \varphi^{p}(\delta) \\
& \geq-n \alpha \varphi^{p}(r) \quad \text { for } 0 \leq r \leq \delta .
\end{aligned}
$$

Since $\varphi$ is in $C^{1}$, we have

$$
\varphi_{r r}+\frac{n-1}{r} \varphi_{r} \geq-\beta \varphi^{p} \text { for } 0 \leq r<1,
$$


in the distribution sense, where

$$
\beta=\max [\alpha((n-2)-\alpha), n \alpha]
$$

We now let $u_{0}(r)=\mu \varphi(r)$, then

$$
\begin{aligned}
& \left(u_{0}\right)_{r r}+\frac{n-1}{r}\left(u_{0}\right)_{r}+\frac{1}{2}\left(\frac{1}{\int_{0}^{1} n \omega_{n}\left(u_{0}\right)^{p} r^{n-1} d r}\right)\left(u_{0}\right)^{p} \\
\geq & (-\mu \beta)\left(\frac{u_{0}}{\mu}\right)^{p}+\frac{1}{2}\left(\frac{1}{\frac{n \omega_{n}}{n-\alpha p}+\sigma_{1}(\delta)}\right)\left(\frac{u_{0}}{\mu}\right)^{p} \\
\geq & \frac{1}{4} \frac{n-\alpha p}{n \omega_{n}} \mu^{-p}\left(u_{0}\right)^{p} \geq\left(u_{0}\right)^{p} \quad \text { for } 0 \leq r<1,
\end{aligned}
$$

provided we take $\mu$ and $\delta$ such that $0<\mu \leq \mu_{0}, 0<\delta<\delta_{0}$ for some sufficiently small $\mu_{0}$ and $\delta_{0}$. We now fix $\mu=\mu_{0}$. Thus $u_{0}(r)$ will be uniquely determined by the choice of $\delta$.

Next, we want to give an estimate for the modulus of continuity of the integral $\int_{B_{1}(0)} u^{p}(x, t) d x$ near $t=0$ which is uniformly valid for small $\delta$. Since we want to prove that the solution blows up in finite time, we can always assume for the contrary that it exists for $0<t<1$. Note that

$$
m=\int_{B_{1}(0)} u_{0}(x) d x=n \mu \omega_{n}\left[\frac{1}{n-\alpha}+\left(\frac{2+\alpha}{2 n}-\frac{\alpha}{2(n+2)}-\frac{1}{n-\alpha}\right) \delta^{n-\alpha}\right] .
$$

It is clear that $m$ is bounded from above and below uniformly for $\delta<\delta_{0}$. By the constraint condition, we know

$$
\int_{B_{1}(0)} u(x, t) d x=t+m
$$

Since $u_{0}(x)=u_{0}(r)$ is monotone decreasing in $r$, applying the maximum principle to $\left(r^{n-1} u_{r}\right)$ gives us $u_{r}(r, t) \leq 0$. Hence

$$
\begin{aligned}
u(r, t) \omega_{n} r^{n}=u(r, t) \int_{0}^{r} n \omega_{n} z^{n-1} d z & \leq \int_{0}^{r} u(z, t) n \omega_{n} z^{n-1} d z \\
& \leq \int_{0}^{1} u(z, t) n \omega_{n} z^{n-1} d z=t+m
\end{aligned}
$$


which implies that,

$$
u(r, t) \leq \frac{t+m}{\omega_{n} r^{n}} \leq \frac{1+m}{\omega_{n} r^{n}} \text { for } 0<t<1
$$

Thus $u(r, t)$ is uniformly bounded, say, in $[1 / 2,1] \times[0,1)$. Next, by Hölder's inequality, one can easily derive

$$
k(t) \leq \frac{w_{n}^{p-1}}{m^{p}} \text { for } 0<t<1
$$

Observe that the function $w(r, t)=r^{n-1} u_{r}$ satisfies the following equation

$$
\mathcal{L}[w]=0 \quad \text { for } 0<r<1,0<t<1
$$

where $\mathcal{L}[\cdot]$ is defined by

$$
\mathcal{L}[\psi]=\psi_{t}-\psi_{r r}+\frac{n-1}{r} \psi_{r}-p k(t) u^{p-1} \psi .
$$

Comparing $w$ with a function $v$ satisfying the same equation $\mathcal{L}[v]=0$, but equals to 0 on $r=1 / 2$ and on $r=1$, and equals to $r^{n-1}\left(u_{0}\right)_{r}(r)$ on $t=0$, we easily obtain that

$$
u_{r}(3 / 4, t) \leq-c_{0} \quad \text { for } 0<t<1
$$

where the constant $c_{0}$ is independent of $\delta$ because of the estimates (4.8) and (4.9).

We next introduce the auxiliary function as in [10],

$$
J=w(r, t)+\varepsilon r^{n} u^{q},
$$

where we fix $q$ such that $q<p$, and $2 p /(q-1)<n$. We assume that (we shall justify this assumption later on)

$$
u(3 / 4, t) \geq \frac{1}{2} u_{0}(3 / 4)
$$

especially, we fix $\eta$ small such that

$$
u(r, t) \geq u(3 / 4, t) \geq \frac{1}{2} u_{0}(3 / 4) \geq \eta \quad \text { for } 0 \leq r \leq 3 / 4,0<t<1 .
$$


A direct calculation shows that

$$
\begin{aligned}
\mathcal{L}[J]= & \varepsilon\left\{-(p+q) k(t) r^{n} u^{p+q-1}\right. \\
& \left.-2 n q u^{q-1} r^{n-1} u_{r}-r^{n} q(q-1) u^{q-2}\left(u_{r}\right)^{2}\right\} \\
= & \varepsilon\left\{-(p+q) k(t) r^{n} u^{p+q-1}-2 n q u^{q-1}\left[J-\varepsilon r^{n} u^{q}\right]-r^{n} q(q-1) u^{q-2}\left(u_{r}\right)^{2}\right\} \\
\equiv & -2 \varepsilon n q u^{q-1} J+\varepsilon c(r, t),
\end{aligned}
$$

where the coefficient of $J$ in the above equation is bounded as long as the solution $u(r, t)$ remains bounded. Since $u(r, t) \geq \eta$,

$$
\begin{aligned}
c(r, t) & =r^{n} u^{2 q-1}\left[-(p+q) k(t) u^{p-q}+2 n \varepsilon q\right]-r^{n} q(q-1) u^{q-2}\left(u_{r}\right)^{2} \\
& \leq r^{n} u^{2 q-1}\left[-(p+q) k(t) \eta^{p-q}+2 n \varepsilon q\right] \\
& <0
\end{aligned}
$$

provided

$$
\varepsilon<\frac{p-q}{2 n q} k(t) \eta^{p-q}
$$

We need a lower bound of $k(t)$ for the above to be valid for small $\varepsilon$. If we assume that

$$
k(t) \geq \frac{1}{2} k(0)
$$

then (4.15) is valid for small $\varepsilon$.

On $\{t=0\}$, for $0<r \leq \delta$, note that $\alpha p=\alpha+2$,

$$
\begin{aligned}
\left(u_{0}\right)_{r}+\varepsilon r\left(u_{0}\right)^{p} & =-\mu \frac{\alpha}{\delta^{\alpha+2}} r+\varepsilon r \mu^{p}\left[\frac{1}{\delta^{\alpha}}\left(1+\frac{\alpha}{2}\right)-\frac{\alpha}{2 \delta^{\alpha+2}} r^{2}\right]^{p} \\
& \leq-\mu \frac{\alpha}{\delta^{\alpha+2}} r+\varepsilon r \mu^{p} \frac{1}{\delta^{\alpha p}}\left(1+\frac{\alpha}{2}\right)^{p} \\
& =\frac{\mu}{\delta^{\alpha+2}} r\left[-\alpha+\varepsilon \mu^{p-1}\left(1+\frac{\alpha}{2}\right)^{p}\right] \\
& <0
\end{aligned}
$$

if $\varepsilon$ is small enough (independent of $\delta$ ). For $\delta<r<1$,

$$
\begin{aligned}
\left(u_{0}\right)_{r}+\varepsilon r\left(u_{0}\right)^{p} & =-\mu \frac{\alpha}{r^{\alpha+1}}+\varepsilon r \mu^{p} \frac{1}{r^{\alpha p}} \\
& =\frac{\mu}{r^{\alpha+1}}\left[-\alpha+\varepsilon \mu^{p-1}\right] \\
& <0,
\end{aligned}
$$


for small $\varepsilon$. Since $p>q$ and $u>\eta$ by the assumption (4.13), it follows that

$$
\left(u_{0}\right)_{r}+\varepsilon r\left(u_{0}\right)^{q}<0 \quad \text { for } 0 \leq r \leq 3 / 4
$$

for $\varepsilon$ small enough. Thus $J<0$ on $\{t=0,0 \leq r \leq 3 / 4\}$. By (4.8) and (4.12), we can choose $\varepsilon$ to be small enough (independent of $\delta$ ) so that $J<0$ on $\{r=$ $3 / 4,0<t<1\}$. We now fix such an $\varepsilon$. Obviously, $J=0$ on $\{r=0,0 \leq t<1\}$. Thus, the maximum principle implies that $J \leq 0$ on $\{0 \leq r \leq 3 / 4,0 \leq t \leq 1\}$, as long as (4.13) and (4.16) remain valid.

Integrating $-u_{r} \geq \varepsilon r u^{q}$ gives us

$$
u^{1-q}(r, t) \geq \frac{q-1}{2} \varepsilon r^{2}+u^{1-q}(0, t) \geq \frac{q-1}{2} \varepsilon r^{2},
$$

i.e.,

$$
u(r, t) \leq\left[\frac{2}{(q-1) \varepsilon}\right]^{q-1} \frac{1}{r^{2 /(q-1)}}=\left[\frac{2}{(q-1) \varepsilon}\right]^{q-1} \frac{1}{r^{\gamma}} \quad \text { for } 0<r<3 / 4,
$$

where $\gamma=2 /(q-1)<n / p$. It follows that

$$
\sup _{0<\delta \leq \delta_{0}} \int_{0}^{\lambda} n \omega_{n} u^{p}(r, t) r^{n-1} d r \leq n \omega_{n}\left[\frac{2}{(p-1) \varepsilon}\right]^{p(q-1)} \frac{\lambda^{n-\gamma p}}{n-\gamma p} .
$$

Since $u(r, t)$ is uniformly bounded in the domain $\{\lambda \leq r \leq 1,0 \leq t \leq 1\}$, the standard parabolic estimates imply that

$$
\lim _{t \rightarrow 0} \sup _{0<\delta \leq \delta_{0}}\left|\int_{\lambda}^{1} n \omega_{n} u^{p}(r, t) r^{n-1} d r-\int_{\lambda}^{1} n \omega_{n}\left(u_{0}\right)^{p} r^{n-1} d r\right|=0
$$

(4.17) and (4.18) imply that

$$
\lim _{t \rightarrow 0} \sup _{0<\delta \leq \delta_{0}}\left|\int_{0}^{1} n \omega_{n} u^{p}(r, t) r^{n-1} d r-\int_{0}^{1} n \omega_{n}\left(u_{0}\right)^{p} r^{n-1} d r\right|=0
$$

Therefore, by the continuation argument in $t$, there exists a $\nu_{0} \in(0,1 / 2)$ such that (4.13) and (4.16) are valid for $0<t<\nu_{0}$, where $\nu_{0}$ is independent of $\delta$. It follows that all of the above estimates are valid for $0<t<\nu_{0}$. 
We now compare $u(r, t)$ with the solution $v(r, t)$ of the following problem

$$
\begin{aligned}
& v_{t}=\Delta v+\frac{1}{2} k(0) v^{p} \quad \text { for } 0 \leq r<1, t>0 \\
& \frac{\partial v}{\partial r}=-c^{*} \quad \text { for } r=1, t>0 \\
& \frac{\partial v}{\partial r}=0 \text { for } r=0, t>0 \\
& v(r, 0)=u_{0}(r) \text { for } 0 \leq r \leq 1
\end{aligned}
$$

where

$$
c^{*}=-\left.\frac{\partial u_{0}}{\partial r}\right|_{r=1} \cdot
$$

By the comparison principle, $u(r, t) \geq v(r, t)$, as long as both solutions exist and $0 \leq t \leq \nu_{0}$. Let $\psi=v_{t}-v^{p}, \frac{\partial \psi}{\partial r}>0$ on $\{r=1, t>0\}$. By using (4.5), we obtain $\psi \geq 0$ on $\{0 \leq r \leq 1, t=0\}$. Thus by using the equation for $\psi$, we can easily obtain that $\psi \geq 0$, as long as the solution exists. Hence

$$
v(r, t) \geq \frac{1}{\left(\frac{1}{u_{0}^{p-1}(r)}-(p-1) t\right)^{1 /(p-1)}} .
$$

Especially,

$$
v(0, t) \geq \frac{1}{\left(\frac{1}{u_{0}^{p-1}(0)}-(p-1) t\right)^{1 /(p-1)}}
$$

Therefore, the solution $v(r, t)$ blows up at a time $T^{*}<\nu$, if $\delta$ is chosen to be small enough. This proves the theorem.

Remark 4.1: For a general function $g(t)$, Theorem 4.1 holds if $g(t) \geq g_{0}>0$ and $g^{\prime}(t) \geq g_{0}>0$ for some positive constant $g_{0}$.

\section{Nonlinear Boundary Value Problem}

In this section we shall generalize the above results into the problem with $f(u, k(t))$ as the boundary function. We first consider the case where $f(u, k(t))=|u|^{p-1} u-$ $k(t)$. 
Consider the following problem:

$$
\begin{aligned}
& u_{t}=\Delta u \text { for } x \in \Omega, t>0 ; \\
& \frac{\partial u}{\partial \nu}=|u|^{p-1} u-k(t) \quad \text { for } x \in \partial \Omega, t \geq 0 ; \\
& u(x, 0)=u_{0}(x) \text { for } x \in \Omega .
\end{aligned}
$$

An additional condition is imposed as follows:

$$
\int_{\Omega} u(x, t) d x=1 \quad \text { for } t \geq 0
$$

We are interested in the case where $p>1$. It is known (cf. [11], etc.) that when $k(t)=0$, the solution will blow up in finite time for any nonnegative $u_{0}(x)$ which is not identically zero. Will the stabilizing factor $k(t)$ on the boundary be able to prevent the blowup phenomenon? We shall answer this question in this section. We shall assume that $u_{0}(x)$ is smooth, say in $C^{3}(\bar{\Omega})$, for convenience. It is clear from the standard theory of parabolic equations that the problem (5.1)-(5.2) has a local solution.

Theorem 5.1: The solution of (5.1)-(5.2) will blow up in finite time if

$$
J(0)=-\frac{1}{2} \int_{\Omega}\left|\nabla u_{0}\right|^{2} d x+\frac{1}{p+1} \int_{\partial \Omega}\left|u_{0}\right|^{p+1} d s
$$

is big enough.

Proof: The proof is similar to that of Theorem 2.1. We multiply the equation (5.1) by $u$ and $u_{t}$, respectively, and integrate over $\Omega$, to get

$$
\begin{aligned}
& \frac{d}{d t}\left(\frac{1}{2} \int_{\Omega} u^{2} d x\right)+\int_{\Omega}|\nabla u|^{2} d x=\int_{\partial \Omega}|u|^{p+1} d s-\frac{1}{|\partial \Omega|} \int_{\partial \Omega}|u|^{p-1} u d s \\
& \int_{\Omega} u_{t}^{2} d x+\frac{d}{d t} \int_{\Omega} \frac{1}{2}|\nabla u|^{2} d x=\frac{d}{d t}\left(\frac{1}{p+1} \int_{\partial \Omega}|u|^{p+1} d s\right) .
\end{aligned}
$$

Let

$$
J(t)=-\frac{1}{2} \int_{\Omega} \nabla u^{2} d x+\frac{1}{p+1} \int_{\partial \Omega}|u|^{p+1} d s
$$

and

$$
I(t)=\int_{0}^{t} \int_{\partial \Omega} u^{2} d s d t+A+B t^{2} .
$$


By a similar calculation, we see that there exists a constant $\alpha>0$ such that

$$
I(t) I^{\prime \prime}(t)-(1+\alpha) I^{\prime}(t)^{2} \geq 0,
$$

provided that $J(0)$ is large enough. We shall not give the detail here.

Next we consider the case where $f(u, k(t))=k(t) u^{p}$ on the boundary. In this case, as the interpolation inequality is different from the previous situation, we have a different inequality about $p$ and $n$ to ensure the existence of a global solution. For simplicity, we again assume that

$$
\int_{\Omega} u(x, t) d x=g(t) \equiv t+m,
$$

where $m=\int_{\Omega} u_{0}(x) d x$. It can be easily seen that the result will be valid for a general bounded smooth function $g(t)>0, g^{\prime}(t)>0$.

Consider the problem:

$$
\begin{aligned}
& u_{t}=\Delta u \quad \text { for } x \in \Omega, t>0, \\
& \frac{\partial u}{\partial \nu}=k(t) u^{p} \quad \text { for } x \in \partial \Omega, t>0, \\
& u(x, 0)=u_{0}(x) \quad \text { for } x \in \Omega .
\end{aligned}
$$

where

$$
k(t)=\frac{1}{\int_{\partial \Omega} u^{p}(x, t) d s} .
$$

We assume that $u_{0}(x)>0$ and $u_{0}(x) \in C^{3}(\bar{\Omega})$. The compatibility is also assumed:

$$
\frac{\partial u_{0}(x)}{\partial \nu}=\frac{u_{0}(x)}{\int_{\partial \Omega} u_{0}(x) d s} \quad \text { for } x \in \partial \Omega .
$$

Again the classical theory of parabolic equations implies the problem (5.5) has a unique classical solution locally in time. Moreover, the strong maximum principle implies that $u(x, t)>0$ for $x \in \bar{\Omega}$ as long as it exists.

Theorem 5.2: For $1<p<(n-1) /(n-2)$, if $n \geq 3$, and $1<p<\infty$, if $1 \leq n \leq 2$, the problem (5.5) has a unique solution for all $0 \leq t<\infty$. 
Proof: The proof is based the similar idea to that of Theorem 3.1. For any $\beta>0$, we multiply the equation by $u^{\beta}$ to obtain

$$
\left(\frac{1}{\beta+1} \int_{\Omega} u^{\beta+1} d x\right)_{t}+\frac{4 \beta}{4(\beta+1)^{2}} \int_{\Omega}\left|\nabla u^{(\beta+1) / 2}\right|^{2} d x=\frac{\int_{\partial \Omega} u^{p+\beta} d s}{\int_{\partial \Omega} u^{p} d s} .
$$

By Hölder's inequality,

$$
\int_{\partial \Omega} u^{p+\beta} d s \leq\left(\int_{\partial \Omega} u^{(p+\beta) a q} d s\right)^{1 / q}\left(\int_{\partial \Omega} u^{(p+\beta)(1-a) q^{*}} d s\right)^{1 / q^{*}}
$$

We choose, for $n \geq 3$ (in the case $n=1$ or $n=2$ we choose $q$ to be large enough),

$$
q>\frac{n-1}{n-2}, \quad q-\frac{n-1}{n-2} \ll 1, \quad(p+\beta) a q=(\beta+1) \frac{n-1}{n-2} .
$$

Then $q^{*} \approx n-1$, and $(p+\beta)(1-a) q^{*} \approx(p-1)(n-1)$. Since $(p-1)(n-1)<p$, we get

$$
(p+\beta)(1-a) q^{*}<p
$$

Therefore

$$
\frac{\int_{\partial \Omega} u^{p+\beta} d s}{\int_{\partial \Omega} u^{p} d s} \leq C\left(\int_{\partial \Omega} u^{(\beta+1) q} d s\right)^{1 / q}
$$

If we define $v(x, t)=u(x, t)^{(\beta+1) / 2}$, then by (5.6),

$$
\left(\frac{1}{\beta+1} \int_{\Omega} v^{2} d x\right)_{t}+\frac{4 \beta}{4(\beta+1)^{2}} \int_{\Omega}|\nabla v|^{2} d x \leq C\left(\int_{\partial \Omega} v^{\frac{2(n-1)}{n-2}} d s\right)^{1 / q}
$$

Now we use the following trace inequality (cf. [16]):

$$
\|w\|_{L^{r}(\partial \Omega)} \leq C\left(\|\nabla w\|_{L^{2}(\Omega)}+\|w\|_{L^{2}(\Omega)}\right)
$$

where

$$
r=\frac{2(n-1)}{n-2} \text { for } n \geq 3, \quad \text { while } r \text { is arbitrary for } n=1 \text { or } 2
$$

Since $q>(n-1) /(n-2)$, we obtain

$$
\left(\int_{\Omega} v^{2} d x\right)_{t}+\int_{\Omega}|\nabla v|^{2} d x \leq C_{\beta}\left(\int_{\Omega} v^{2} d x+1\right) .
$$


Using Gronwall's inequality, we have

$$
\begin{gathered}
\left(\int_{\Omega} u^{\beta+1} d x\right)_{t}+\left(\int_{\partial \Omega} u^{(\beta+1) \frac{n-1}{n-2}} d x\right)^{\frac{n-2}{n-1}} \leq C_{\beta}, \\
\sup _{0 \leq t \leq T} \int_{\Omega} u^{\beta+1}(x, t) d x+\int_{0}^{T}\left(\int_{\partial \Omega} u^{(\beta+1) \frac{n-1}{n-2}} d x\right)^{\frac{n-2}{n-1}} d t \leq C_{\beta},
\end{gathered}
$$

where the constant $C$ depends only on the known data and $T$. We fix $\beta$ so that $\beta+1>n(p-1)$, then by Theorem 1.1 in [12],

$$
\sup _{0 \leq t \leq T} \sup _{x \in \bar{\Omega}} u(x, t) \leq C .
$$

Thus by Theorems 7.1-7.2 in Chapter V of [13] (it is clear the assumptions (7.4)(7.6) are satisfied), we immediately obtain that

$$
\|u\|_{C^{1+\alpha,(1+\alpha) / 2}\left(\bar{Q}_{T}\right)} \leq C
$$

for some $\alpha \in(0,1)$. This estimate implies that the function $k(t) u^{p}$ is uniformly bounded in the space $C^{1+\alpha,(1+\alpha) / 2}$. Consequently, we can use Schauder's estimate to obtain

$$
\|u\|_{C^{2+\alpha, 1+(\alpha / 2)}\left(\bar{Q}_{T}\right)} \leq C .
$$

With the above a priori estimates in hand, we can obtain the existence for $0 \leq t \leq$ $T$, for any $T>0$.

Remark 5.1: Similar to Theorem 3.2, the above argument works for the critical number $p=(n-1) /(n-2)$ provided $\inf _{0 \leq t \leq T}\|u\|_{L^{p}(\partial \Omega)}(t)$ is large enough, which is the case if we assume that $\min _{x \in \bar{\Omega}} u_{0}(x)$ to be large enough. Global existence is guaranteed in this case.

Remark 5.2: We conjecture that the solution of (5.1)-(5.2) will blow up in finite time if $p>(n-1) /(n-2)$ and the initial value satisfies a certain condition.

Acknowledgment: The authors would like to thank Professor A. Friedman for some helpful discussion during the preparation of the manuscript. The authors also thank Dr. Henghui Zhou for the partial motivation of the present research. 


\section{References}

[1] J. Bebernes, A. Bressan And A. LACEY, Total blowup versus single point blowup, J. Diff. Equations, 73(1988), 30-44.

[2] J. Bebernes and David Eberly, Mathematical Problems from Combustion Theory, Applied Mathematical Sciences 83, Springer-Verlag, New York, Inc. 1989.

[3] C. BUdd, B. Dold AND A. STUART, Blowup in a partial differential equation with conserved first integral, SIAM J. Applied Math., 53(1993), 718-742.

[4] J. R. Cannon, The One Dimensional Heat Equations, Addison-Wesley, Menlo Park, 1984.

[5] J. R. Cannon, P. DuChateau, K. Steube, Identifying a time-dependent unknown coefficient in a nonlinear heat equation, in Nonlinear Diffusion Equations and their Equilibrium States, 3, ed., N. G, Lloyd, W. M. Ni, L. P. Peletier, J. Serrin, Page 153-170, Birkhauser, Boston, 1992.

[6] J. R. Cannon AND H. M. Yin, A class of nonlinear nonclassical parabolic problems, Journal of Differential Equations, 79(1989), 266-288.

[7] J. M. Chadam, A. Perice AND H. M. Yin, The blowup property of solutions to a chemical diffusion equation with localized reactions, J. Math. Anal. Appl., 169(1992), 313-328.

[8] J. R. ChADAM AND H. M. Yin, An iteration procedure for a class of integrodifferential equations of parabolic type, J. of Integral Equations and Applications, 2(1989), 31-47.

[9] D. Colton, R. Ewing AND W. Rundell, Inverse problems in partial differential equations, SIAM Press, Philadelphia, 1990.

[10] A. Friedman AND B. MCLeOd, Blowup of positive solutions of semilinear heat equations, Indiana Univ. Math. J. 34(1985), 425-477. 
[11] BEI HU AND H.M. YIN, The profile near blowup time for solution of the heat equation with a nonlinear boundary condition, Transaction of American Mathematical Society, submitted.

[12] R. JENSEN AND W. LiU, An $L^{\infty}$ estimate for the heat equation with a nonlinear boundary condition and its applications, Preprint.

[13] O.A. Lady̌̆enskaja, V. A. Solonnikov and N. N. URal'Ceva, Linear and Quasi-linear Equations of Parabolic Type, AMS Trans. 23, Providence., R.I., 1968.

[14] H. A. LEVINE, The role of critical exponents in blowup theorems, SIAM Review, 32(1990), 262-288.

[15] H. A. Levine And L. E. Payne, Nonexistence Theorems for the heat equation with nonlinear boundary conditions and for the porous medium equation backward in time, J. of Diffs. Eqs., 16(1974), 319-334.

[16] V. G. Maz'Ja, Sobolev Spaces, Springer-Verlag, Berlin Heidelberg, 1985.

[17] C.V. Pao, Nonlinear Parabolic and Elliptic Equations, Plenum Press, New York, 1992. 